$\xi=-1$

\title{
PAPR reduction for FBMC/OQAM using hybrid scheme of different Precoding transform and mu-law companding
}

\author{
Imad A Shaheen ${ }^{1 *}$, Abdelhalim zekry ${ }^{1}$, Fatma newagy ${ }^{1}$, Reem Ibrahim ${ }^{2}$ \\ ${ }^{I}$ Electronics and Communication Department, Faculty of Engineering, Ain Shams University, Cairo-Egypt \\ ${ }^{2}$ Embedded System Design Electronics Factory Department Cairo-Egypt \\ *Corresponding author E-mail: imadshaheen@gmail.com
}

\begin{abstract}
The filter banks multicarrier with offset quadrature amplitude modulation (FBMC/OQAM) is developing multicarrier modulation technique used in the next wireless communication system (5G). FBMC/OQAM supports high data rate and high band width efficiency. However, one of the major drawbacks of FBMC system is high peak to Average Power Ratio (PAPR) of the transmitted signal, which causes serious degradation in performance of the system. Therefore, it is required to use a proper PAPR scheme at the transmitter to reduce the PAPR. In this paper, a hybrid scheme is investigated with the combination of preceding transform technique and Mu Law Companding technique to reduce PAPR in FBMC systems. Moreover, four preceding techniques are examined to find the best Precoding technique which can be used with Mu law commanding. We assessed the discrete Hartley transform (DHT). The discrete cosine transformed (DCT), the Discrete Sine Transform (DST), and the Walsh Hadamard transforms (WHT) which are applied separately with Mu Companding. The numerical results verify that the FBMC systems with all Precoding technique combined with Mu law commanding can improve PAPR performance of the signals greatly with the best results achieved when the combination scheme consists of the DST Precoding and Mu law commanding for both PAPR and BER performance.
\end{abstract}

Keywords: FBMC/OQAM; PAPR; 5G; Precoding; Mu-Law Companding.

\section{Introduction}

Multicarrier techniques have been used to support high data rate transmission for wireless communication. The orthogonal frequency division multiplexing (OFDM) technique is one of the most types of multicarrier modulation scheme used for wireless communication.However, the use of the cyclic prefixes $(\mathrm{CP})$ with the OFDM symbol causes reduced spectral efficiency and reduced performance of the system. To overcome these disadvantages of OFDM systems, the filter banks multicarrier with offset quadrature amplitude modulation (FBMC/OQAM) technique has been established as a potential candidate multicarrier modulation scheme for the fifth generation $(5 \mathrm{G})$ wireless communication to support a high spectral efficiency and high data rate [6][10].The high peak-to-average power ratio (PAPR) issue has appeared in all sorts of multicarrier communication systems, which caused serious degradation in performance from the system. Therefore, it is necessary to reduce it by using a proper reduction scheme at the transmitter.

In the literature, there have been several researchers focusing on the PAPR reduction of FBMC/OQAM systems [6-13][2526].Active constellation extensions (ACE) with projection onto convex sets (POCS) are used to reduce PAPR in FBMC system but it reduced BER performance [6]. PAPR reduction schemes originated on Active Constellation Extension (ACE), and tone reservation (TR) is presented [7]. The PTS [8], SLM [9], Potencies of trellis-based SLM [10] and Multi-Block Joint Optimization technique [11] are found to be effective in PAPR reduction. However, they require additional processing and often an increased implementation complexity of the system. Clipping and filtering techniques improve the PAPR, but they fail in BER [12], requiring advanced noise cancellation technique at the receiver

In [13], the authors utilized the sliding window algorithm to improve the tone reservation (TR) scheme and it is acceptable for the PAPR reduction of FBMC/OQAM signal. The advanced technique based on Sliding window tone reservation, the SW-TR, cancel the peaks of the FBMC/OQAM.

In [6], and [7], it has shown that the $\mathrm{Mu}$-law companding schemes can reduce the PAPR in FBMC system better than the clipping approach but $\mathrm{Mu}$-law transform still has a larger average power level and also still exhibits non-uniform distributions [8], and [9]

In this paper, we proposed PAPR reduction schemes for FBMC system by using the combination of Precoding transform techniques and Mu-Law Companding technique. Therefore, we evaluate four types of Precoding technique namely the discrete Hartley transform (DHT), the discrete cosine transform (DCT), the Discrete Sine Transform (DST) and the Walsh Hadamard transform(WHT) which are applied separately to the proposed system with $\mathrm{Mu}$ companding to find the best combination scheme to reduce the PAPR for FBMC. The rest of paper is organized as follows: in section II, FBMC/OQAM system model is introduced, in section III, the PAPR theory in FBMC system is described, then in section IV, different Precoding transform techniques areexplained,in section V Mu-law companding scheme is described, and in section VI the simulation results are presented. Last section VIII, concludes the paper 


\section{FBMC/OQAM system model}

In this section, the functions performed by the transmitter and receiver of the system will be mathematically modeled according to the system building blocks shown in Figure 1. The first function to be performed in the transmitter is to precode K QAM symbols in parallel. The output of the precoder will be phase rotated by multiples of pi/2 sequentially. The output signal vector is then transformed to the time domain by IFFT operation. The resulting $\mathrm{K}$ samples will be filtered by a filter bank for pulse shaping. The signal is then converted from parallel to serial form and compressed according to a standard compression law. The receiver performs the inverse operation to retrieve the QAM symbols from the received noisy and distorted signal. The inverse of the pulse shaping filter bank is a matched filter bank.

In the proposed FBMC system the information symbols are processed with $K$ is total subcarriers used in FBMC system and $M$ is time slots. FBMC/OQAM system is to transmit offset QAM symbols. The complex QAM data symbol $s_{k, m}$ with $k t h$ subcarrier and mth time slot can be expressed as:

$S_{k, m}=S_{k, m}^{R}+j S_{k, m}^{I}$

Where $s_{k, m}^{R}$ is the real part of the complex data symbol and $s_{k, m}^{I}$ is its imaginary part.To enable offset QAM modulation the real and imaginary parts of complex QAM symbols $a_{K, M}$ are separated and arranged in a $K \times 2 M$ matrix $A$ as follows:

$A=\left[\begin{array}{ccc}a_{0,0} & a_{0,1} & a_{0,2 M-1} \\ a_{1,0} & a_{1,1} & a_{1,2 M-1} \\ a_{K-1,0} & a_{K-1,1} & a_{K-1, M-1}\end{array}\right]$

(2) And the prototype filter coefficients vector as

Where $a_{K, M}$ denoted the transmitted QAM symbol, and A matrix can be rewritten to enable OQAM as follows

$$
A=\left[\begin{array}{llll}
\mathrm{s}_{0,0}^{R} & \mathrm{~s}_{0,0}^{I} & \mathrm{~s}_{0,2 \mathrm{M}-1}^{R} & \mathrm{~s}_{0,2 \mathrm{M}-1}^{I} \\
\mathrm{~S}_{1,0}^{R} & \mathrm{~S}_{1,0}^{I} & \mathrm{~s}_{1,2 \mathrm{M}-1}^{R} & \mathrm{~s}_{1,2 \mathrm{M}-1}^{I} \\
\mathrm{~s}_{\mathrm{k}-1,0}^{R} & \mathrm{~S}_{\mathrm{k}-1,0}^{I} & \mathrm{~s}_{\mathrm{k}-1,2 \mathrm{M}-1}^{R} & \mathrm{~s}_{\mathrm{k}-1,2 \mathrm{M}-1}^{I}
\end{array}\right]
$$

The Precoding transform is applied to each column of matrix A, as

$$
\mathrm{y}_{k}=\mathscr{a}_{\mathrm{m}}^{\mathscr{O}}=\mathrm{P}_{\mathrm{K}} \mathrm{a}_{\mathrm{m}},
$$

Where $P_{K}$ is a $\mathrm{K} \times \mathrm{K}$ Precoding matrix which used to spread the energy of symbols over the subcarriers assigned to the user and $a_{m}$ is the mth column of $A$.

Precoding matrix can be written as

$$
\mathrm{P}_{K}=\left[\begin{array}{ccc}
\mathrm{P}_{00} & \ldots \cdots & \mathrm{P}_{0(\mathrm{~K}-1)} \\
\mathrm{P}_{10} & \ldots \cdots & \mathrm{P}_{1(\mathrm{~K}-1)} \\
& & \\
\mathrm{P}_{(\mathrm{K}-1) 0} & \cdots \cdots & \mathrm{P}_{(\mathrm{K}-1)(\mathrm{K}-1)}
\end{array}\right]
$$

(We will discuss in next section), $\mathrm{a}_{\mathrm{m}}$ is the mth column of A. In the OQAM, the phase offsets are introduced as

$$
\mathrm{b}_{\mathrm{m}}=\mathrm{J}_{\mathrm{m}} \mathrm{d}_{\mathrm{m}} \%
$$

Where $\mathrm{J}_{\mathrm{m}}=\operatorname{diag}\left(\left[j^{m}, j^{m+1}, \ldots \ldots \ldots . . j^{m+k-1}\right]\right)$ then the transmitted precode FBMC signal is given as

$$
\begin{aligned}
& x[n]=\sum_{\mathrm{k}=0}^{K-1} \sum_{m=0}^{2 M-1} \mathrm{j}^{k+m} \mathscr{Z}_{\mathrm{k}, \mathrm{m}} \mathrm{g}[(\mathrm{n}-\mathrm{mK} / 2) K M] \mathrm{e}^{\mathrm{j} \frac{2 \pi \mathrm{kn}}{\mathrm{K}}}, \\
& x[n]=\sum_{\mathrm{k}=0}^{K-1} \sum_{m=0}^{2 M-1} \mathrm{j}^{k+m} \mathscr{Z}_{k, \mathrm{~m}} g_{k, m}[n]
\end{aligned}
$$

Where

$$
g_{k, m}[n]=\mathrm{g}[(\mathrm{n}-\mathrm{mK} / 2) K M] \mathrm{e}^{\mathrm{j} \frac{2 \pi k n}{K}}
$$

Where, and $\mathscr{G}_{k, \mathrm{~m}}$ is the $k t h$ element of $\mathscr{O}_{m}^{c}$, and $g[n]$ is the impulse response of the prototype filter with coefficients $K M$ and the length of prototype filter as

$$
L=K M-1
$$

The filter must satisfy the orthogonal condition restricted to the real field

$\mathfrak{R}\left\{\sum_{\mathrm{n}=-\infty}^{+\infty} \mathrm{g}_{\mathrm{k}^{\prime}, m^{\prime}}[\mathrm{n}] \mathrm{g}_{\mathrm{k}, m}^{*}[\mathrm{n}]\right\}=\delta_{\mathrm{k}, \mathrm{k}^{\prime}} \delta_{m, m^{\prime}}$

Where $\delta_{\mathrm{i}, j}$ is the Kroneckerdelta and $\mathfrak{R}$ stands for the real part.

We used the matrix form to describe the PolyPhase structure, thus let us denote the transmitted vector as

$$
x=[\mathrm{x}[0], \mathrm{x}[1], \mathrm{x}[2], \ldots \ldots \ldots, \mathrm{x}[\mathrm{KM}-1]]^{\mathrm{T}}
$$

$\mathrm{g}=[\mathrm{g}[0], \mathrm{g}[1], \ldots ., \mathrm{g}[\mathrm{KM}-1]]^{\mathrm{T}}$

Then we can rewrite the equation of Precoding FBMC/OQAM signal as

$$
x[n]=\sum_{m=0}^{2 M-1} G_{m} F_{K}^{H} b_{m}
$$

Where $F_{K}^{H}$ is $\mathrm{K}$ point IFFT matrix In the above equation, $b_{m}$, the baseband signal in frequency domain, is first transformed into the time domain by multiplying it with inverse FFT matrix $F_{K}^{H}$. Then multiplying it by PolyPhase filter $G_{m}$.finally, we obtain the transmitted Precoding FBMC/OQAM signal by summing all pulse shaped sub-symbol vectors. Since $\mathrm{b}_{\mathrm{m}}=\mathrm{J}_{\mathrm{m}} \mathscr{\mathrm { d }}_{\mathrm{m}}^{o}=J_{m} P_{k} a_{m}$. Equation (14) can be put in the form

$x[n]=\sum_{m=0}^{2 M-1} G_{m} F_{K}^{H} J_{m} P_{k} a_{m}$

Then the Precoding FBMC signal $x_{k}$ will be fed to the companding block so the output of the companding is expressed as

$\mathrm{S}[n]=\mathrm{C}\{\mathrm{x}[\mathrm{n}]\}$

Where $C[]$ is companding function which applied at end of the transmitter side (explain in next section) 


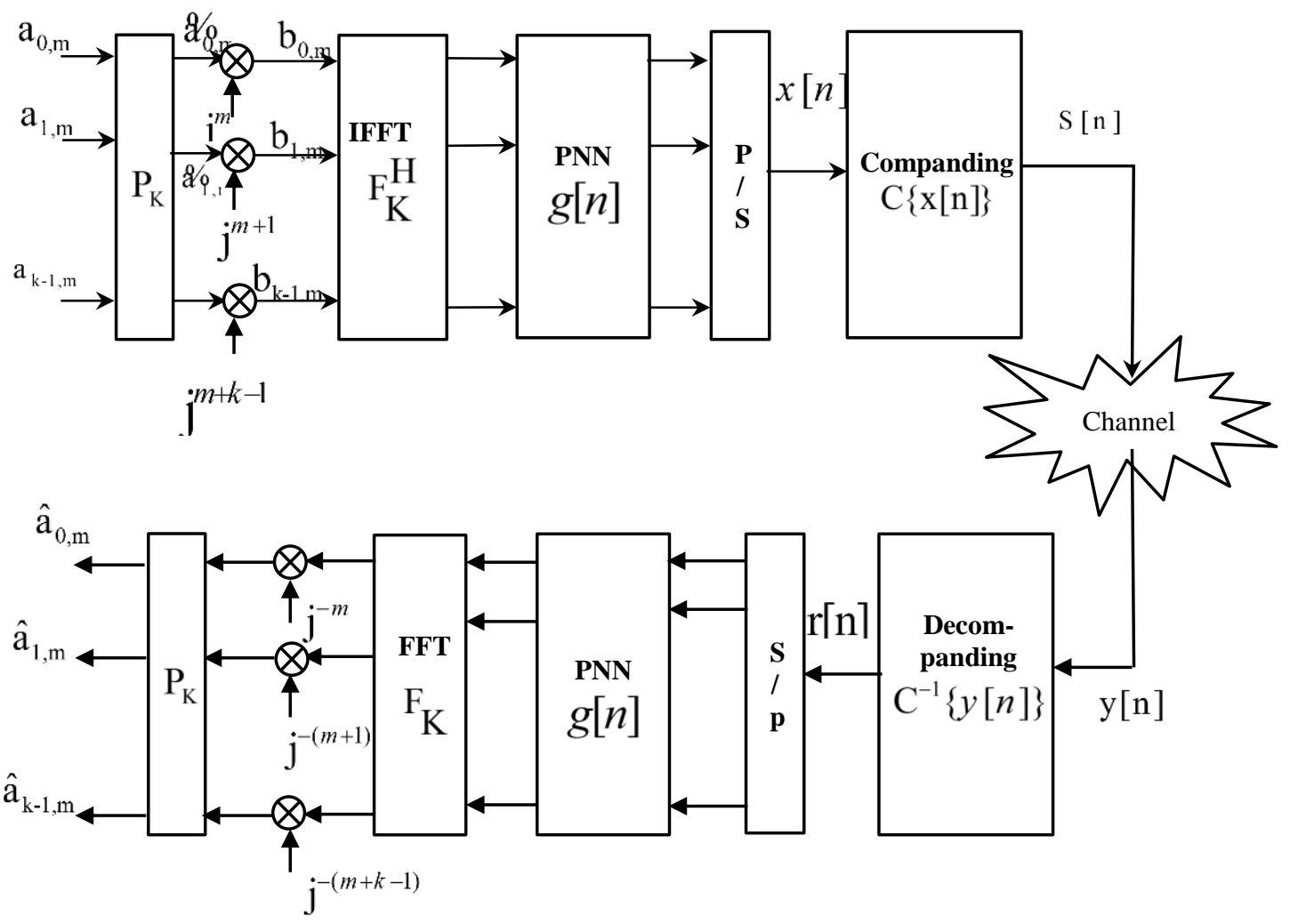

Fig. 1: FBMC/OQAM System with Precoding transform and Companding Technique.

Then the discrete transmitted Precoding FBMC/OQMA system with companding transform is expressed as

$\mathrm{s}[n]=\sum_{m=0}^{2 M-1} C G_{m} F_{K}^{H} J_{m} P_{k} a_{m}$

Then the received signal can be written as

$y[n]=h[n] * s[n]+\eta[n]$

Where $\eta[n]$ is a vector of the additive white Gaussian noise (AWGN) and * is the convolution operator, and $h[n]$ is the channel impulse response for a particular channel realization.

The recovered signal can be expressed as

$r[n]=C^{-1}(y[n])$

Where $C^{-1}$ is the de-companding $\mathrm{Mu}$ process, after the demodulation, the received signal can be expressed as

$r_{k, m}=\Re\left\{\sum_{n=1}^{L} g_{k, m}^{*}[n] r[n] \mid+\eta_{k, m}\right\}$

$r_{k, m}=\hat{r}_{k, m}+\mathfrak{R}\left\{\eta_{k, m}\right\}$

where $\hat{r}_{k, m}$ Is the useful signal

$\eta_{k, m}=\sum \eta[n] g_{k, m}[n]$

Where

$$
g_{k, m}[n]=\mathrm{g}[(\mathrm{n}-\mathrm{mK} / 2) K M] \mathrm{e}^{\mathrm{j} \frac{2 \pi k n}{K}}
$$

By putting equ(19) in eq (18) we will get

$$
\eta_{k, m}=\sum_{n} \eta[n] \mathrm{g}[(\mathrm{n}-\mathrm{mK} / 2) K M] \mathrm{e}^{\frac{j 2 \pi k n}{K}}
$$

(17) Since we recover the $q_{k, \mathrm{~m}}$ thanks to the real orthogonality then $\eta_{k, m}$ can be written as follows

Where $\eta_{k, m}$ is a linear combination of Gaussian random variables

The Gaussian random variance can be expressed as

$\eta_{k, m}=\sum_{n=m \frac{K}{2}}^{L-1+m \frac{K}{2}} \eta[n] \mathrm{g}[(\mathrm{n}-\mathrm{mK} / 2) K M] \mathrm{e}^{-\mathrm{j} \frac{2 \pi k n}{K}}$

$\sigma_{\eta_{t, m}}^{2}=\sigma_{\eta}^{2} \sum_{n=m \frac{K}{2}}^{L-1+m \frac{K}{2}} \mathrm{~g}[(\mathrm{n}-\mathrm{mK} / 2) K M]=\sigma_{\eta}^{2}$

Where the normalization of the prototype filters implies

$\sum_{n=m \frac{K}{2}}^{L-1+m \frac{K}{2}} \mathrm{~g}[(\mathrm{n}-\mathrm{mK} / 2) K M]=1$

Since the Precoding matrix $\mathrm{P}$ is a unitary matrix and if there is no ISI and ICI the noise variance will not change neither after signal fed to FFT nor after the signal fed to the PolyPhase filter.

Finally after the de-precoding transform. The output symbol for the mth time slot is given by

$\hat{a}_{m}=P_{K}^{H} C^{-1} \mathfrak{R}\left\{G_{m} F_{K} J_{m}^{H}\right\}$

Where $P_{k}^{\mu}$ de-Precoding process at the receiver side is, $F_{K}$ is FFT process, $G_{m}$ is apolyphasefilterand $\mathfrak{R}\left\{G_{m} F_{K} J_{m}^{H}\right\}$ is the real part of OQAM transmitted symbols. To guarantee perfect reconstruction in FBMC, The combined response of the transmit filter and received filter must be Nyquist pulse thus we used the square root 
raised cosine (SRRC) filter to design a PolyPhase with length of filter $L=K M$ where the coefficients are real and symmetric such that

$$
g[n]=g[K M-n]
$$

We chose the prototype filter, using SRRC filter where the frequency response of SRRC filter is defined by

$$
\mathrm{g}(\mathrm{f})=\left\{\begin{array}{cc}
T & \text { for } 0 \leq|\mathrm{f}| \leq \frac{1-\mathrm{r}}{2 \mathrm{~T}} \\
\frac{\mathrm{T}}{2}\left[1+\cos \frac{\pi \mathrm{T}}{\mathrm{r}}\left\{|\mathrm{f}|-\frac{1-\mathrm{r}}{2 \mathrm{~T}}\right\}\right] & \text { for } \frac{1-\mathrm{r}}{2 \mathrm{~T}} \leq|\mathrm{f}| \leq \frac{1+\mathrm{r}}{2 \mathrm{~T}} \\
0 & \text { for }|\mathrm{f}| \geq \frac{1+\mathrm{r}}{2 \mathrm{~T}}
\end{array}\right.
$$

Where Tis symbol period and the symbol rate is $f=1 / T$. Where $r$ is the roll-offParameter $0 \leq \mathrm{r} \leq 1$,however, the impulse response of the SRRC filter in the continuous time domain is expressed as [11] [12].

$$
g(t)=\frac{\sin \left((1-r) \frac{\pi t}{T}\right)+\frac{4 r t}{T} \cos \left((1+r) \frac{\pi t}{T}\right)}{\frac{\pi t}{T}\left(1-\left(\frac{4 r t}{T}\right)^{2}\right)}
$$

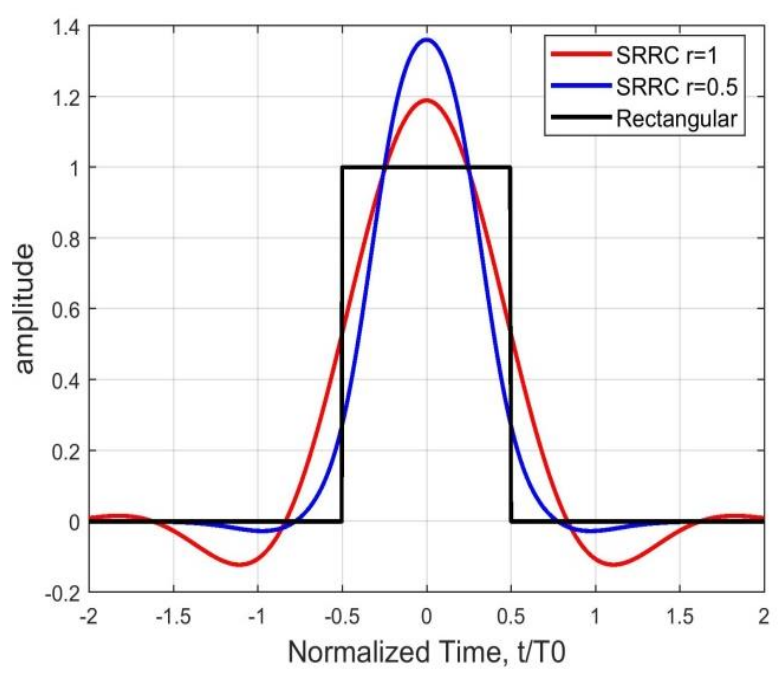

Fig. 2: The Impulse Responses of SRRC Filter with Various Roll-Off Factors.

Fig. 2 gives the SRRC representation with roll-off factor equal $r=$ 1 and $r=0.5$ in frequency response; we observe that the SRRC prototype presents a significant improvement compared to the rectangular window prototype in terms of frequency selectivity

\section{The Peak to Average Power Ratio (PAPR)}

The Peak to Average Power Ratio (PAPR) of FBMC/OQAM signal $\mathrm{s}_{\mathrm{n}}$ is described as the ratio of the peak power of $x[n]$ to its average power. The PAPR of FBMC/OQAM transmitted signal can be written as [21][23-24]

PAPR $=10 \log _{10} \frac{\operatorname{Max}\left\{|x[n]|^{2}\right\}}{\mathrm{E}\left\{|x[n]|^{2}\right\}} \mathrm{dB}$

Where $E\{$.$\} expresses the expectation operation. Additionally, for$ the FBMC/OQAM signals, the complementary cumulative distribution function (CCDF) of PAPR giving the probability $P_{r}$ that the
PAPR is above several threshold levels $(\gamma)$ can be expressed by [22]:

$$
\operatorname{CCDF}(\gamma)=\operatorname{P}_{r}(\operatorname{PAPR}(x[n])>\gamma)=1-\left(1-\mathrm{e}^{-\gamma}\right)^{k}
$$

\section{Precoding techniques}

Before the FBMC modulation, each of data FBMC block is multiplied by a Precoding matrix to the input symbols which is known as data-independent process of Precoding. The Precoding technique is used to reduce the autocorrelation of the input sequence to reduce the peak to average power (PAPR) of FBMC signal and the results make the envelope almost constant.It also works with a random number of subcarriers and it can improve the BER performance of FBMC signals.

However, there are some important conditions for using Precoding matrix to reduce PAPR:

I). the Precoding matrix must be an orthogonal matrix P by satisfying the condition

$$
P^{*} P=I
$$

Where $P^{*}$ means Hermitian transpose of the matrix $\mathrm{P}$, and I means the identity matrix

II). all singular values of Precoding matrix P must be equal to one to obtain minimum BER

\subsection{Types of Precoding matrix}

We assessed four types of Precoding transforms which are applied to the system separably to measure the performance of each technique combined with Mu companding.

\subsubsection{Discrete cosine transforms (DCT)}

Mathematically, the unitary Discrete Cosine Transform (DCT) of an input sequence $a_{m}$ with length Nis given by the following formula

$$
\mathrm{y}_{k}=\mathrm{T}_{\mathrm{k}} \sum_{\mathrm{k}=0}^{\mathrm{N}} \mathrm{a}_{m} \cos \left[\frac{\pi(2 \mathrm{n}+1) \mathrm{k}}{2 \mathrm{~N}}\right] \quad \mathrm{k}=1,2, \ldots ., N-1
$$

Where the analysis window $T_{k}$ can be expressed as:

$$
\mathrm{T}_{\mathrm{k}}= \begin{cases}\frac{1}{\sqrt{\mathrm{N}}} & , k=1 \\ \frac{2}{\sqrt{\mathrm{N}}} & , 2 \leq k \leq N\end{cases}
$$

Where $\mathrm{a}_{m}$ is the input to the Precoding transform, $y_{k}$ is the output of Precoding transform

\subsubsection{The Walsh Hadamard Transforms}

The Walsh Hadamard Transform (WHT) is a non-sinusoidal and can be implemented by a butterfly structure as in FFT. This means that applying WHT does not require the extensive increase in system complexity. WHT decomposes a signal into a set of basic functions. These functions are Walsh functions, which are square waves with values of +1 or -1 [17]. The proposed Hadamard transform scheme may reduce the occurrence of the high peaks comparing the original FBMC system. The kernel of WHT can be written as follows:

$\mathrm{H}_{1}=[1]$ 


$$
\begin{aligned}
& \mathrm{H}_{2}=\frac{1}{\sqrt{2}}\left[\begin{array}{cc}
1 & 1 \\
1 & -1
\end{array}\right] \\
& \mathrm{H}_{2 N}=\frac{1}{\sqrt{2 \mathrm{~N}}}\left[\begin{array}{cc}
\mathrm{H}_{\mathrm{N}} & \mathrm{H}_{\mathrm{N}} \\
\mathrm{H}_{\mathrm{N}} & -\mathrm{H}_{N}
\end{array}\right]
\end{aligned}
$$

The output sequence of WHT can be written as:

$\mathrm{y}_{k}=\frac{1}{N} \sum_{\mathrm{k}=0}^{N-1} a_{\mathrm{m}} \operatorname{WAL}(n, \mathrm{k})$

Where $\mathrm{k}=0,1, \ldots ., \mathrm{N}-1$ WAL $(n, \mathrm{i})$ are Walsh functions, $\mathrm{a}_{m}$ is the input to the Precoding transform, $y_{n}$ is the output of Precoding transform

\subsubsection{The Discrete Sine Transforms}

The output sequence of a DST Precoding with length $\mathrm{N}$ can be written as

$$
\mathrm{y}_{K}=\sum_{\mathrm{k}=0}^{\mathrm{N}-1} \mathrm{a}_{m} \sin \left[\pi \frac{k n}{\mathrm{~N}+1}\right] \quad \mathrm{k}=1,2, \ldots \ldots, N-1
$$

Where $\mathrm{a}_{m}$ is the input to the Precoding transform, $y_{k}$ is the output of Precoding transform

\subsubsection{The discrete Hartley transform}

The output sequence of a DHT with length $\mathrm{N}$ can be written as

$\mathrm{y}_{k}=\sum_{\mathrm{k}=0}^{\mathrm{N}-1} \mathrm{a}_{\mathrm{m}}\left[\cos \frac{2 \pi \mathrm{nk}}{\mathrm{N}}+\sin \frac{2 \pi \mathrm{nk}}{\mathrm{N}}\right] \quad \mathrm{k}=1,2, \ldots \ldots, N-1$

For, where $\mathrm{a}_{m}$ is the input to the Precoding transform, $y_{k}$ is the output of Precoding transform

\section{Non-linear companding technique}

It is one of the most attractive schemes due to its good system performance, BER, low implementation complexity and absence of bandwidth expansion. This transform technique performs compression at the transmitter end after synthesis filter bank and expansion at the receiver end before the signal is fed to analysis filter. It uses a strict monotonically increasing function for companding. Therefore; the companded signal at the transmitter can be correctly recovered at the receiver using an inverse transform function Companding increases the SNR when the input signal is low and therefore it reduces the effect of systems noise source

\subsection{Mu law companding technique}

Figure 3 shows the Mu-law compressor characteristics with different values of $\mathrm{Mu}$ ratio which is used to control the amount of companding in the FBMC signal. From the figure we can observe that when the MU ratio increases it results in more compression for the higher input values, while if $\mathrm{Mu}=1$, no compression occurs.

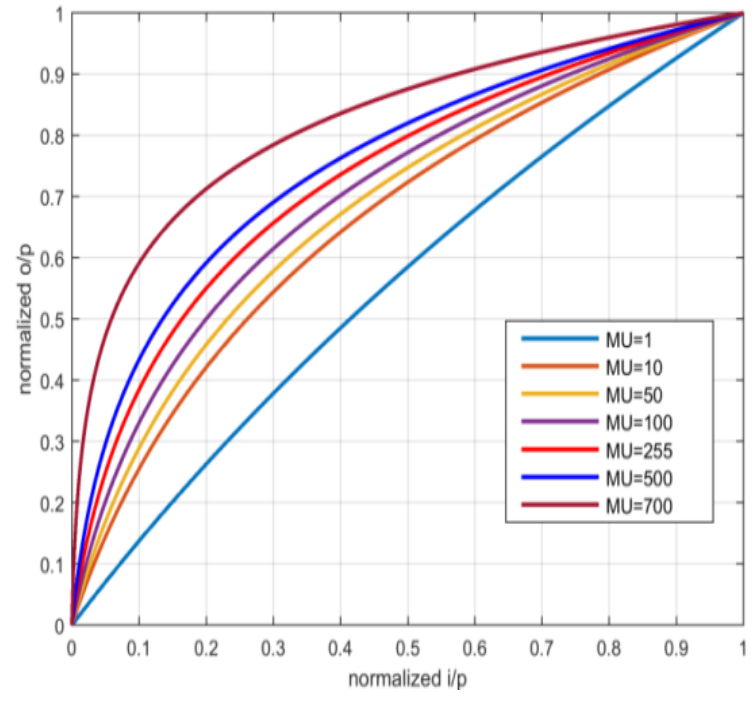

Fig. 3: Mu-Law Compressor Characteristics.

The companding function applied to the end of the transmitter side is expressed by [21-22]

$s[n]=\mathrm{C}(x[n])=\operatorname{sgn}(x[n]) \frac{\ln (1+\mathrm{Mu}|x[n]|}{\ln (1+\mathrm{Mu})}$,

Where $\mathrm{Mu}$ parameter used to control the companding level applied to the signal. The inverse companding at the receiver is given by

$r[n]=F^{-1}(y[n])=\operatorname{sgn}(y[n])\left(\frac{1}{M u}\right)\left((1+M u)^{|y \cdot n| \mid}-1\right)$,

Where $r_{k}$ is receives signal, $\mathrm{Mu}$ is the ratio of companding, and sgn is assign of signal.

\section{Simulation result}

Extensive simulations are conducted to assess the performance of the proposed scheme. We designed the FBMC/OQAM with 256 subcarriers and oversampling factor $\mathrm{L}=4$. In our design, the prototype filter is the square root raised cosine filter SRRC with roll factor of 0.5 and length of 4T. For the PAPR of FBMC hybrid schemes are implemented with different Precoding techniques and Mu Law companding scheme.

Table 1: Simulation Parameters of the Proposed System

\begin{tabular}{ll}
\hline Parameter & Value \\
\hline Total number of subcarriers & 256 \\
Data blocks & 2000 \\
Modulation & OQAM \\
Overlapping factor & 4 \\
Roll factor & 0.5 \\
SNR range & $0-25$ \\
Mu-ratio & 255
\end{tabular}




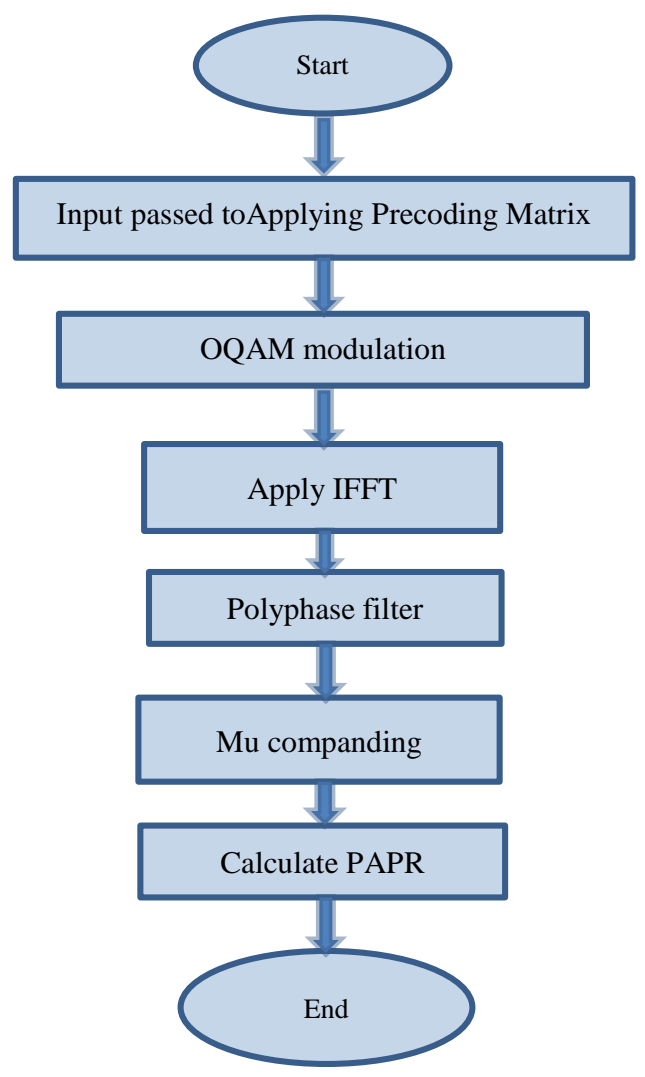

Fig. 4: Flow Chart of Proposed Hybrid Scheme.

The simulation results were obtained by considering an Additive White Gaussian Noise (AWGN) channel. Fig 4 shows the flow chart of processes for FBMC/OQAM with Precoding and Companding. The simulation parameters for FBMC/OQAM system which is used to analyze the PAPR and BER performance of the proposed hybrid scheme is presented in Table I

\subsection{Performance in PAPR reduction}

In FBMC/OQAM systems, the complementary cumulative distribution function (CCDF) is used to evaluate PAPR reduction performance, which denotes the probability that the PAPR exceeds a certain threshold. For a given threshold, a lower CCDF indicates better PAPR performance. We have applied 2000 random FBMC blocks to obtain the CCDFs of different signals.

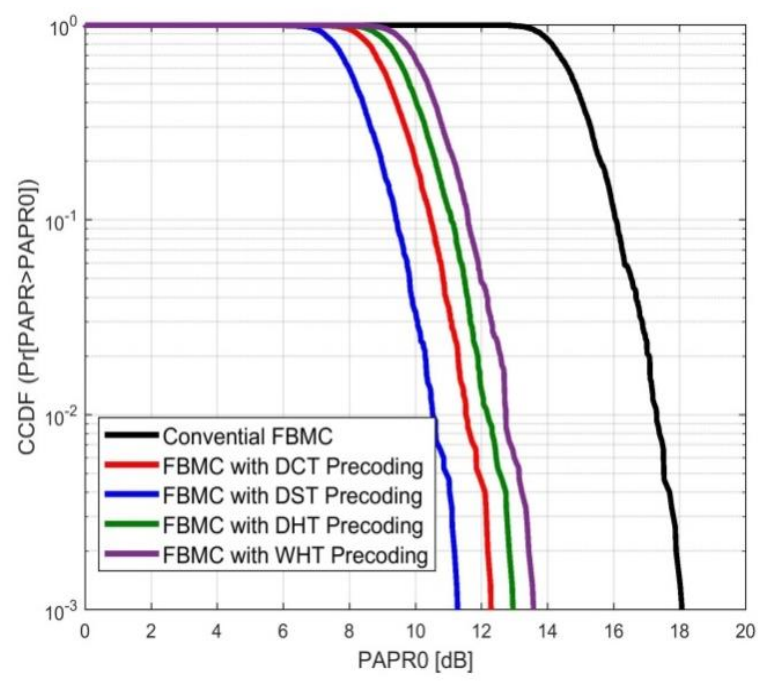

Fig. 5: FBMC/OQAM systems with Using Precoding Transform Technique.
Fig.5 Illustrates the CCDFs of conventional FBMC/OQAM system and different Precoding transform techniques which are applied separately to the proposed scheme to assess theperformance of each of them. Clearly, we observe that all Precoding techniques have much lower PAPR than the conventional FBMC/OQAM signal. For example, when $\mathrm{CCDF}=10^{-3}$, the PAPR for the conventional FBMC signals is $18.00 \mathrm{~dB}$, whereas that of the DST precoding signal is $11.23 \mathrm{~dB}$, with an improvement of $6.77 \mathrm{~dB}$. However, from Fig. 5 and TableII, we can observe that The DST gives better performance than other precoding techniques

Table 2: PAPR Reduction for FBMC/OQAM with Using Different Precoding Technique

\begin{tabular}{ll}
\hline Scheme & PAPR dB \\
\hline Conventional FBMC & 18.00 \\
FBMC with DHT Precoding & 13.31 \\
FBMC with DCT Precoding & 13.02 \\
FBMC with DST Precoding & 11.23 \\
FBMC with WHT Precoding & 13.49 \\
\hline
\end{tabular}

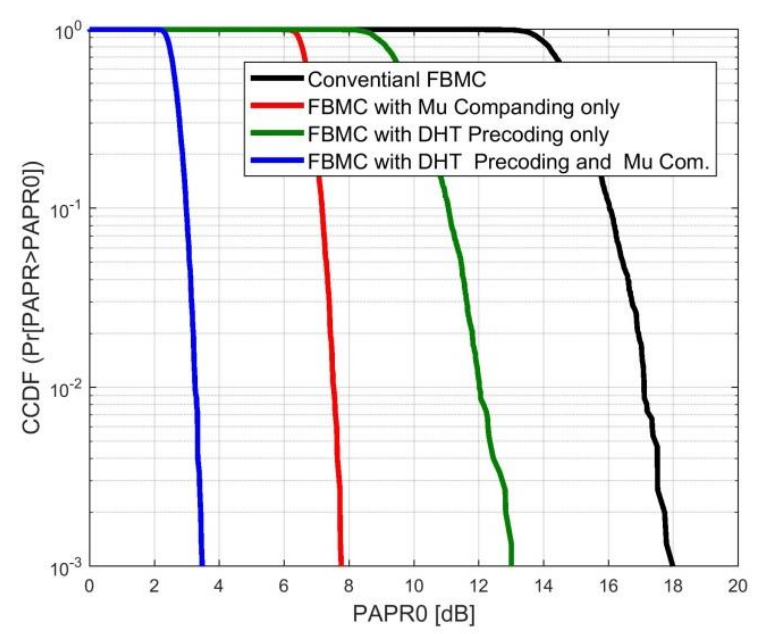

Fig. 6: FBMC/OQAM System with Hybrid Scheme Consist From Discrete Hartley Transform (DHT) and Mu Law Companding.

Table 3: PAPR Reduction for FBMC/OQAM with Using Discrete Hartley Transform and Mu Law

\begin{tabular}{ll}
\hline Scheme & PAPR dB \\
\hline Conventional FBMC & 18.00 \\
FBMC with Mu Companding only & 7.74 \\
FBMC with DHT Precoding only & 13.31 \\
FBMC with DHT Precoding and Mu Comp. & 3.45 \\
\hline
\end{tabular}

Fig.6 Illustrates the CCDFs of conventional FBMC/OQAM signal, Mu Law companding signal ,the Discrete Hartley Transform (DHT) Precoding signal, and Hybrid scheme consisting of the Discrete Hartley Transform (DHT) Precoding with Mu Law companding technique for reducing the PAPR in FBMC/OQAM system with companding factor $\mathrm{Mu}=255$.

Table III depicts the PAPR at $\mathrm{CCDF}=10^{\wedge}-3$ for the abovementioned schemes in the figure. Clearly, from figure 6 and table III, we observe that, when we use the DHT Precoding with Mucompanding, The PAPR appreciably improves. At clip rate of $10^{-3}$, the PAPR is reduced to $3.45 \mathrm{~dB}$ which is really an enormous improvement.

Table 4: PAPR Reduction for FBMC/OQAM with Using Discrete Cosine Transform (DCT) and Mu Law Companding

\begin{tabular}{ll}
\hline Scheme & PAPR dB \\
\hline Conventional FBMC & 18.00 \\
FBMC with Mu Companding & 7.899 \\
FBMC with DCT Precoding & 13.02 \\
FBMC with DCT Precoding & 3.536 \\
\hline
\end{tabular}




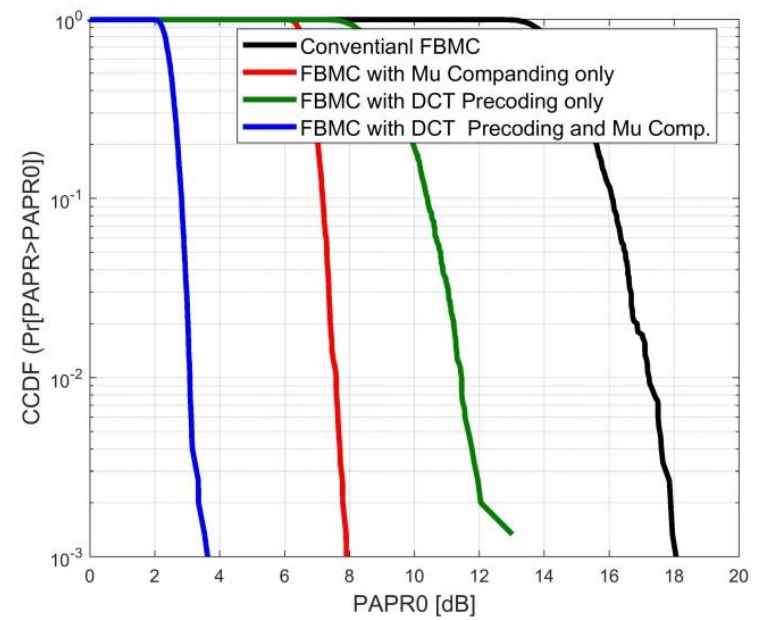

Fig. 7: FBMC/OQAM system with Hybrid Scheme Consist from Discrete Cosine Transform and Mu Law Companding.

Fig.7 Illustrates the CCDFs of conventional FBMC/OQAM signal, Mu Law companding signal, the discrete cosine transform (DCT) Precoding signal and Hybrid scheme consisting of the discrete cosine transform Precoding with Mu Law companding technique for reducing the PAPR in FBMC/OQAM system.

Table IV depicts the PAPR at $\mathrm{CCDF}=10^{\wedge}-3$ for the different schemes in Fig. 7.Clearly, from figure 7 and table IV, we observe that, when we use the DCT Precoding with Mu-companding, the PAPR is improving. At clip rate of $10^{-3}$, the PAPR is reduced to the $3.536 \mathrm{~dB}$ which is a considerable achievement.

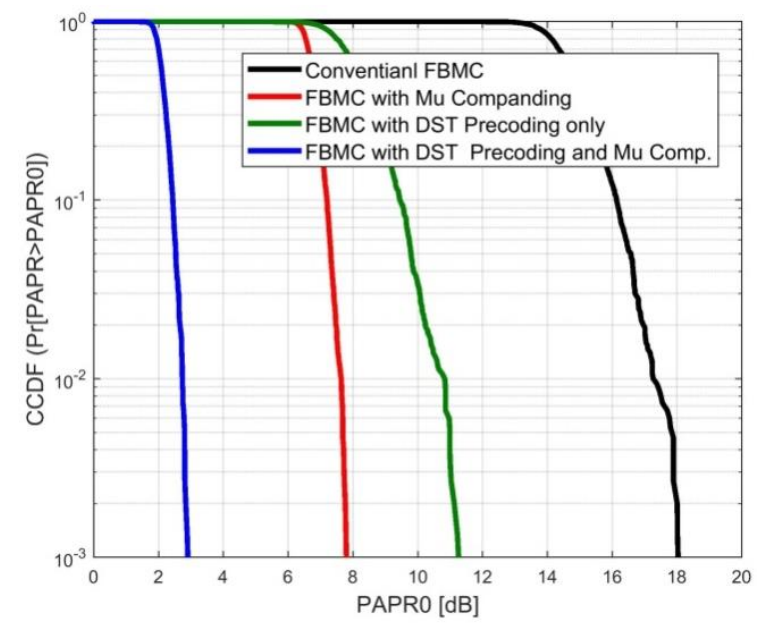

Fig. 8: FBMC/OQAM System with Hybrid Scheme Consist from Discrete Sine Transform and Companding.

Fig.8 Illustrates the CCDFs of conventional FBMC/OQAM signal, $\mathrm{Mu}$ Law companding signal, the discrete sine transform (DST) Precoding signal, and Hybrid scheme consisting of the discrete sine transform (DST) Precoding with Mu Law companding technique for reducing the PAPR in FBMC/OQAM system.

Table V depicts the PAPR at $\mathrm{CCDF}=10^{\wedge}-3$ for the same schemes in Fig. 8.Clearly, from figure 8 and table $\mathrm{V}$, we observe that when we used the DST Precoding with Mu-companding, the PAPR further improves. At clip rate of $10^{-3}$, the PAPR is reduced to the $2.888 \mathrm{~dB}$ when is even better than the previous techniques

Table 5: PAPR Reduction for FBMC/OQAM with Using Discrete Sine transform and Mu Law Companding

\begin{tabular}{ll}
\hline Scheme & PAPR dB \\
\hline Conventional FBMC & 18.00 \\
FBMC Mu Companding only & 7.790 \\
FBMC with DST Precoding only & 11.23 \\
FBMC with DST Precoding and Mu Comp. & 2.888 \\
\hline
\end{tabular}

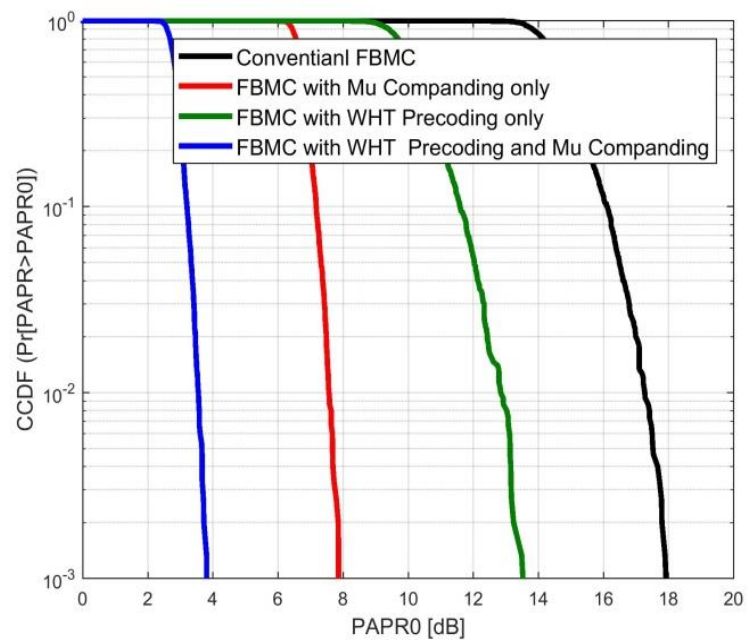

Fig. 9: FBMC/OQAM with Hybrid Scheme Consist from Walsh Hadamard Transform and Companding.

Table 6: PAPR Reduction for FBMC/OQAM with Using Walsh Hadamard Transform and Mu Law Companding

\begin{tabular}{ll}
\hline Scheme & PAPR dB \\
\hline Conventional FBMC & 18.00 \\
FBMC with Mu Companding only & 7.866 \\
FBMC with WHT only & 13.49 \\
FBMC with WHT Precoding and Mu Comp. & 3.807 \\
\hline
\end{tabular}

Fig.9 Illustrates the CCDFs of conventional FBMC/OQAM signal, Mu Law companding signal, the Walsh Hadamard Transform (WHT) Precoding signal and Hybrid scheme consisting of the Walsh Hadamard Transform (WHT) Precoding with Mu Law companding technique to reduce the PAPR in FBMC/OQAM system.

Table VI depicts the PAPR at $\mathrm{CCDF}=10^{\wedge}-3$ for the different schemes. Clearly, from figure 9 and table VI, we observe that when we use the WHT Precoding with Mu-companding, The PAPR improves. At clip rate of $10^{-3}$, the PAPR is reduced to $3.807 \mathrm{~dB}$ which is worse than the DST plus the $\mathrm{u}$ law compression. Fig.10 Illustrates the CCDFs of conventional FBMC/OQAM signal, and different hybrid schemes investigated to reducePAPR. Table VII depicts the PAPR at $\mathrm{CCDF}=10^{\wedge}-3$ for the different schemes. Clearly, from figure 10 and table VII, we can observe that a hybrid scheme consisting of the Precoding with $\mathrm{Mu}$ companding for reducing PAPR in the FBMC system can be used to reduce the PAPR with the best result of PAPR reduction can be achieved when we use a combination of the DST with Mu Law companding.

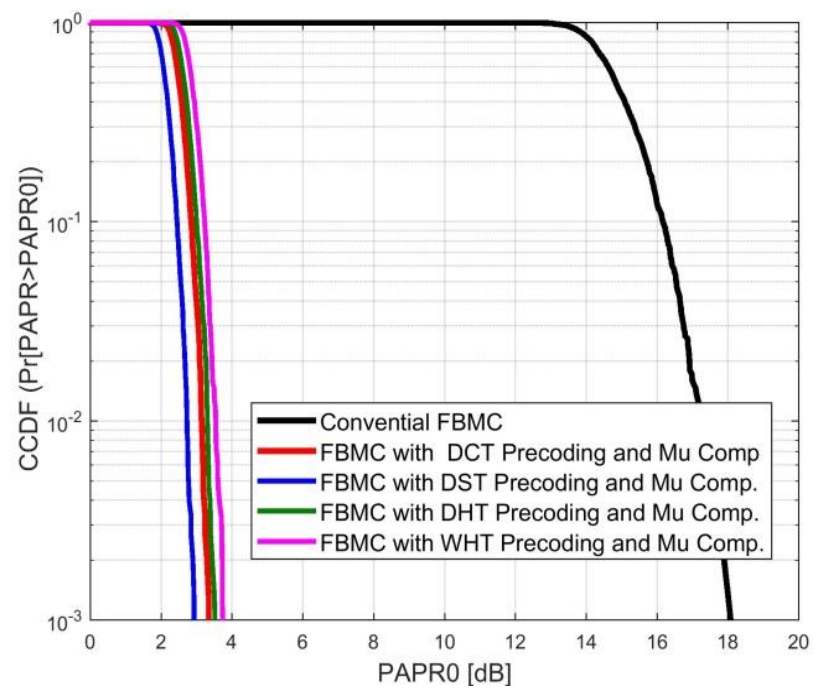

Fig. 10: FBMC/OQAM signal With Hybrid Scheme Consist from Different Precoding and Mu Law Companding. 


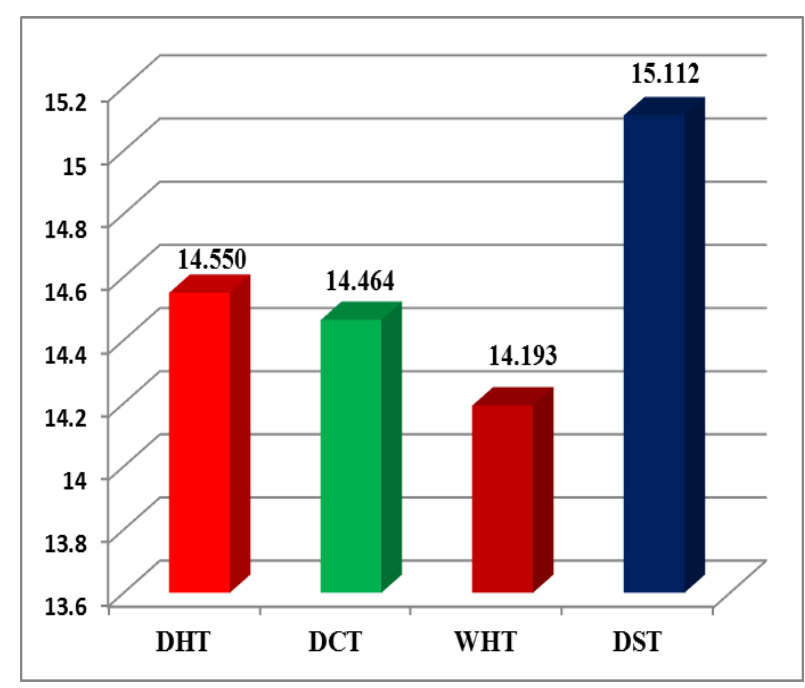

Fig. 11: Bar Chart Analysis of PAPR Using Different Proposed Schemes

Fig. 11 shows the value of PAPR reduction in $\mathrm{dB}$, which proves that for FBMC systems, different Precoding transform combined with $\mathrm{Mu}$ law companding can be used for effective PAPR reduction. We can observe that the combination of DHT with Mu law Companding can reduce the PAPR by approximately $14.55 \mathrm{~dB}$, the combination of DCT with $\mathrm{Mu}$-Law Companding can reduce the PAPR by approximately $14.464 \mathrm{~dB}$, the combination of WHT with Mu law Companding can reduce the PAPR by approximately $14.193 \mathrm{~dB}$ and finally, the combination of DST with Mu law Companding can reduce the PAPR by approximately $15.112 \mathrm{~dB}$.

\subsection{BER performance}

Fig. 12 illustrates the BER of the proposed system as functionof signal to noise ratio for various schemes over an AWGN channel. We can observe that fortunately that the hybrid scheme based on the combination of DST and Mu-Law companding has better performance than other types of hybrid schemes.

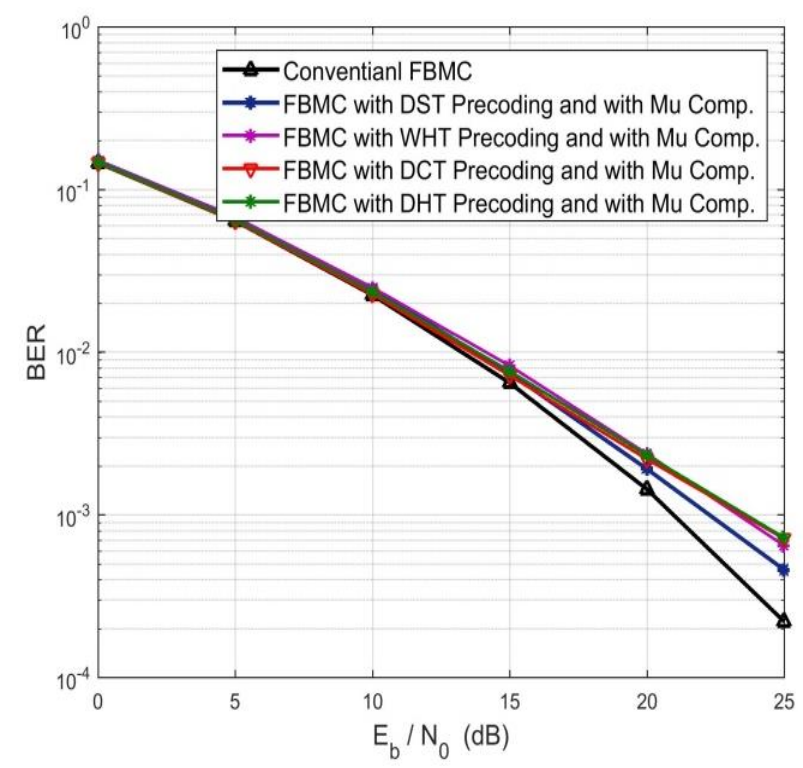

Fig. 12: BER Performance of FBMC/OQAM with Various PAPR Reduction Techniques.

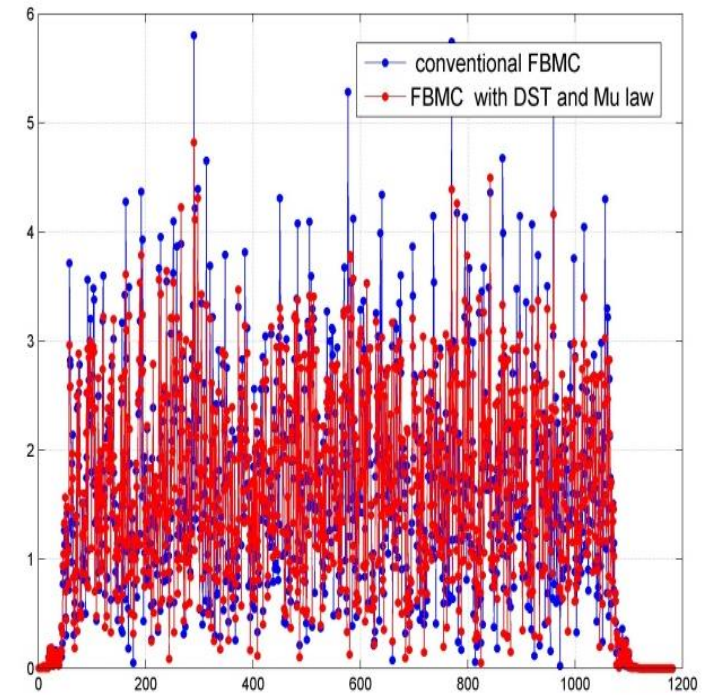

Fig. 13: Peak Canceling Signal of the Conventional FBMC and Hybrid Scheme Consist from DST Precoding and Mu Comp.

Figure 13 illustrates that the time variations of the of the conventional FBMC signal are much larger than that of FBMC with Hybrid scheme from DST Precoding and Mu law Companding which demonstrates the effective PAPR reduction with such hybrid scheme.

\section{Conclusion}

In this paper, we analyzed the performance of FBMC/OQAM system in terms of PAPR reduction by using some preceding techniques combined with $\mathrm{Mu}$-Law commanding.From our simulation, it is found that the hybrid techniques produced the lower PAPR compared to conventional FBMC/OQAM or when using preceding or Mu law commanding technique separately.

Furthermore, hybrid scheme consisting from the DST preceding and $\mathrm{Mu}$ Law commanding produced better results than other hybrid schemes. By using preceding technique, there will be no signal degradation and no need for any side information to send to the receiver side

Moreover, the BER performance of FBMC/OQAM slightly degrades with using the hybrid scheme of any sort of precoding and Mu-Law companding. The implementation complexity of the proposed technique is acceptable since it does not require any optimization from one FBMC block to the next.

\section{Further research}

We intend to include the other types of Precoding like zadoffChu sequence and wavelet transform with other companding techniques as well as using Precoding transform with SLM technique.

\section{References}

[1] R. Nissel, S. Schwarz and M. Rupp, "Filter Bank Multicarrier Modulation Schemes for Future Mobile Communications," in IEEE Journal on Selected Areas in Communications, vol. 35, no. 8, pp. 1768-1782, Aug. 2017.https://doi.org/10.1109/JSAC.2017.2710022.

[2] J. G. Andrews, S. Buzzi, W. Choi, S. V. Hanly, A. Lozano, A. C. Soong, and J. C. Zhang, "What will 5G be?" IEEE Journal on Selected Areasin Communications, vol. 32, no. 6, pp. 1065-1082, 2014. https://doi.org/10.1109/JSAC.2014.2328098.

[3] G. Wunder, P. Jung, M. Kasparick, T. Wild, F. Schaich, Y. Chen,S. Brink, I. Gaspar, N. Michailow, A. Festag et al., "5GNOW: nonorthogonal, asynchronous waveforms for future mobile applications,"IEEE Communications Magazine, vol. 52, no. 2, pp. 97-105, 2014 https://doi.org/10.1109/MCOM.2014.6736749. 
[4] P. Banelli, S. Buzzi, G. Colavolpe, A. Modenini, F. Rusek, andA Ugolini, "Modulation formats and waveforms for $5 \mathrm{G}$ networks: Who will be the heir of OFDM? An overview of alternative modulation schemes for improved spectral efficiency," IEEE Signal Process. Mag.,vol. 31, no. 6, pp. 80-93, 2014. https://doi.org/10.1109/MSP.2014.2337391.

[5] Shaheen, Imad A., et al. "Proposed New Schemes to Reduce PAPR for STBC MIMO FBMC systems." simulation 6.9 (2017). https://doi.org/10.5120/cae2017652556

[6] Imad Ahmed Shaheen, Abdelhalim Zekry, Fatma Newagy and Reem Ibrahim, Egypt. "Combined DHT Precoding and A-Law Companding for PAPR Reduction in FBMC/OQAM signals." International Journal of Computing Academic Research (IJCAR) 6.Number 2 (2017): pp-31.

[7] H. B"olcskei, "Orthogonal frequency division multiplexing based on offset QAM," in Advances in Gabor analysis. Springer, 2003, pp 321-352.

[8] B. Farhang-Boroujeny, "Filter bank multicarrier modulation: A wave-form candidate for $5 \mathrm{G}$ and beyond," Advances in Electrical Engineering, vol. 2014, December 2014

[9] M. G. Bellanger, "Specification and design of a prototype filter for filterbank based multicarrier transmission," in IEEE International Conferenceon Acoustics, Speech, and Signal Processing, vol. 4 2001, pp. 2417-2420.https://doi.org/10.1109/ICASSP.2001.940488

[10] P. Siohan, C. Siclet, and N. Lacaille, "Analysis and design ofOFDM/OQAM systems based on filterbank theory," IEEE Transactionson Signal Processing, vol. 50, no. 5, pp. 1170-1183, 2002 https://doi.org/10.1109/78.995073.

[11] M. Fuhrwerk, J. Peissig, and M. Schellmann, "On the design of an FBMC based air interface enabling channel adaptive pulse shaping persub-band," in IEEE Signal Processing Conference (EUSIPCO), 2015,pp. 384-388 https://doi.org/10.1109/EUSIPCO.2015.7362410

[12] M. Caus and A. I. P'erez-Neira, "Transmitter-receiver designs for highlyfrequency selective channels in MIMO FBMC systems," IEEE Transactions on Signal Processing, vol. 60, no. 12, pp. 65196532, 2012. https://doi.org/10.1109/TSP.2012.2217133.

[13] S. Mirabbasi and K. Martin, "Design of prototype filter for nearperfect-reconstruction overlapped complex-modulated transmultiplexers," in IEEE International Symposium on Circuits and Systems, 2002. https://doi.org/10.1109/ISCAS.2002.1009967.

[14] C. Y. Hsu and H. C. Liao, "PAPR reduction using the combination of precoding and Mu-Law companding techniques for OFDM systems," 2012 IEEE 11th International Conference on Signal Processing, Beijing, 2012, pp. 1-4 https://doi.org/10.1109/ICoSP.2012.6491517.

[15] R. Chandrasekhar, M. Kamaraju, M. V. S. Sairam and G. T. Rao, "PAPR reduction using combination of precoding with Mu-Law companding technique for MIMO-OFDM systems," 2015 International Conference on Communications and Signal Processing (ICCSP), Melmaruvathur, 2015, pp. 0479-0483. https://doi.org/10.1109/ICCSP.2015.7322430.

[16] M. J. Hao and C. H. Lai, "Precoding for PAPR Reduction of OFDM Signals With Minimum Error Probability," in IEEE Transactions on Broadcasting, vol. 56, no. 1, pp. 120-128, March 2010. https://doi.org/10.1109/TBC.2009.2034512.

[17] B. Farhang-Boroujeny, "OFDM versus filter bank multicarrier," IEEE Signal Processing Mag., vol. 28, no. 3, pp. 92-112, May 2011. https://doi.org/10.1109/MSP.2011.940267.

[18] S. A. Aburakhia, E. F. Badran and D. A. E. Mohamed, "Linear Companding Transform for the Reduction of Peak-to-Average Power Ratio of OFDM Signals," in IEEE Transactions on Broadcasting, vol. 55, no. 1, pp. 155-160, March 2009 https://doi.org/10.1109/TBC.2009.2013987.

[19] N. Varghese, J. Chunkath and V. S. Sheeba, "Peak-to-Average Power Ratio Reduction in FBMC-OQAM System," 2014 Fourth International Conference on Advances in Computing and Communications, Cochin, 2014, pp. 286-290. https://doi.org/10.1109/ICACC.2014.74

[20] B. Farhang-Boroujeny and C. H. (George) Yuen, "Cosine modulated and offset QAM filter bank multicarrier techniques: A continuous-ime prospect," EURASIP Journal on Advances in Signal Processing, $\quad$ vol. $2010, \quad$ pp. $1-17, \quad 2010$ https://doi.org/10.1155/2010/165654.

[21] N. Raju and S. S. Pillai, "Companding and Pulse shaping technique for PAPR reduction in FBMC systems," 2015 International Conference on Control, Instrumentation, Communication and Computational Technologies (ICCICCT), Kumaracoil, 2015, pp. 89-93 https://doi.org/10.1109/ICCICCT.2015.7475255.

[22] N. Raju and S. S. Pillai, "Companding and Pulse shaping technique for PAPR reduction in FBMC systems," 2015 International Con- ference on Control, Instrumentation, Communication and Computational Technologies (ICCICCT), Kumaracoil, 2015, pp. 89-93. https://doi.org/10.1109/ICCICCT.2015.7475255.

[23] I. A. A. Shaheen, A. Zekry, F. Newagy and R. Ibrahim, "Absolute Exponential Companding to Reduced PAPR for FBMC/OQAM," 2017 Palestinian International Conference on Information and Communication Technology (PICICT), Gaza City, 2017, pp. 60-65. https://doi.org/10.1109/PICICT.2017.17.

[24] I. A. Shaheen, A. Zekry, F. Newagy and R. Ibrahim, "Modified Square Rooting Companding Technique to Reduced PAPR for FBMC/OQAM," 2017 Palestinian International Conference on Information and Communication Technology (PICICT), Gaza City, 2017, pp. 66-70. https://doi.org/10.1109/PICICT.2017.18.

[25] Zhu, X., Zhu, G. and Jiang, T. (2009), 'Reducing the peak-toaverage power ratio using unitary matrix transformation', Communications, IET, 3,161-171.https://doi.org/10.1049/iet-com:20080194

[26] Wang, C.-L., Wang, S.-S., Chen, H.-M. "An improved metric-based active constellation extension scheme for PAPR reduction in OFDM systems" IEEExplore (2016), 978-1-5090-0314-3/16, 4p https://doi.org/10.1109/WTS.2016.7482033 